Baltic Astronomy, vol.9, 395-402, 2000.

\title{
CCD PHOTOMETRY OF VARIABLE SUBDWARFS AND WHITE DWARFS AT CALAR ALTO OBSERVATORY
}

Sonja Schuh ${ }^{1}$, Stefan Dreizler ${ }^{1}$, Jochen L. Deetjen ${ }^{1}$, Uli Heber ${ }^{2}$ and Ralf D. Geckeler ${ }^{3}$

1 Institut für Astronomie und Astrophysik, Waldhäuser Strasse 64, D72076 Tübingen, Germany

2 Remeis-Sternwarte Bamberg, Sternwartstrasse 7, D-96049 Bamberg, Germany

3 Institut für Astrophysik, Technikerstrasse 25, A-6020 Innsbruck, Austria

Received December 20, 1999

Abstract. We report on our time-resolved photometry of subdwarfs and white dwarfs at the Calar Alto Observatory. Candidates are taken from a spectroscopically pre-selected sample obtained from the Hamburg Schmidt Survey. We also report on our experience during the WET run in April 1999 and introduce our revised CCD photometry reduction package TRIPP, as well as preliminary results.

Key words: stars: subdwarfs, white dwarfs, oscillations

\section{INTRODUCTION}

Asteroseismology is the only method to directly probe the stellar structure. Under certain conditions part of the thermal energy of a star is efficiently transformed into mechanical energy providing a continuous driving of pulsations. These pulsations can be observed due to periodic changes in the surface brightness. The frequencies of the observed eigenmodes directly constrain the physical properties of the stellar structure. This method therefore is a very valuable tool to test stellar evolution models.

In principle, pulsating white dwarfs and B subdwarf stars (sdB) are ideal seismological objects since they exhibit several excited modes. In the case of white dwarfs, the pulsations are non-radial 
$g$-modes. Variable hydrogen-rich white dwarfs are found around an effective temperature of $12000 \mathrm{~K}$ (spectral type DA, variable type DAV or ZZ Ceti) whereas pulsating hydrogen-poor white dwarfs are found among the DB white dwarfs between 20000 and $25000 \mathrm{~K}$ or among the C-He-O rich PG 1159 stars in the range from $70000 \mathrm{~K}$ up to $140000 \mathrm{~K}$ (DOV or GW Vir stars). The recently discovered pulsating sdB stars (EC 14026 stars, Kilkenny et al. 1997) populate the very hot end of the horizontal branch and show $p$-mode and/or $g$-mode pulsations. The periods in these evolved stars are of the order of minutes and therefore easily observable. With non-radial pulsations, small deviations from spherical symmetry, e.g. rotation or magnetic fields, can be studied. The detection limits are far below the spectroscopic ones. The simultaneous excitation of several modes also allows a precise determination of global stellar parameters like mass and luminosity independently from spectroscopic methods. Therefore a number of pulsating white dwarfs are intensively observed in single site observations and in worldwide coordinated campaigns. One of these campaigns was XCOV17, the Whole Earth Telescope (WET) run in April 1999, in which we participated using the onsite CCD camera (instead of a standard WET photometer). In the northern hemisphere, XCOV17 concentrated on the EC 14026 variable PG 1336-018, for results see other articles in these proceedings. However, despite the very high scientific potential of white dwarf asteroseismology, only few objects are known where the full potential of this approach can be exploited. To improve this situation we have been observing a sample of spectroscopically pre-selected hot white dwarfs and subdwarfs. Time-resolved photometric monitoring of the objects allows a reliable classification as stable or pulsating, which will hopefully provide us with more good asteroseismologic objects as the project goes on. The candidates found to be variable so far are presented here.

\section{OBSERVATIONS}

\subsection{Telescope and instrumentation used in XCOV17}

During the WET XCOV17 campaign, and also for most observations related to the search of new variable white dwarfs and subdwarfs, we used the DSAZ (Calar Alto) $1.23 \mathrm{~m}$ telescope (located in southern Spain, aberration-free field of view of $15^{\prime}$ ). 
The detector is a CCD camera equipped with a blue-sensitive SITe chip. As most standard instrumentation, this camera has not been specifically designed to serve as fast photometry device. In order to obtain sufficient time resolution, we binned the exposures and made use of windowing, reading out only part of the chip. A compromise between the lower readout times for smaller windows and the decreasing number of comparison stars available in the field yields acceptable sampling time intervals and satisfying $\mathrm{S} / \mathrm{N}$ at the same time. Using a self-developed software tool, which allows for uninterrupted exposure series, we achieve cycle times (interval between start of two subsequent exposures) down to around 30 seconds.

\subsection{Objects observed within our project and XCOV17}

Table 1 lists the candidates from our project sample that have already been observed and gives an idea of the respective time bases of the observations by means of the number of frames taken in a given night. The total size of our sample (Heber et al. 1999) contains more than a hundred stars, and follow-up observations have already partly been granted observing time.

In the WET run, PG 1336-018 was observed from Calar Alto in the nights from $02 / 04$ to $13 / 04$ with the exception of $12 / 04$, with an average of 750 frames per night. PG 1159-035 was monitored from $02 / 04$ to $05 / 04$ with roughly 250 frames per night, Feige 48 on 11/04 and 12/04 (831 and 985 frames).

\section{SOFTWARE: TRIPP}

The reduction of CCD images requires several steps which will be described in the following. For our completely new version of the software we started from the "CCD-package" developed by R. Geckeler in IDL. Both the old package and the new revision called TRIPP (Time Resolved Imaging Photometry Package) are available from the authors upon request.

\subsection{Data reduction}

The bias and flatfield corrections are prepared for by generating a median bias and flatfield image from the calibration images. 
Table 1. Overview of observed objects

\begin{tabular}{|c|c|c|c|}
\hline Object & Spectral type & Date & \# Frames \\
\hline \multicolumn{4}{|l|}{ DSAZ $2.2 \mathrm{~m}$} \\
\hline Abell 43 & PG 1159 & $27 / 05 / 98$ & 489 \\
\hline PG $1144+005$ & PG 1159 & $26 / 05 / 98$ & 112 \\
\hline HS $1831+6432$ & $\mathrm{sdB}$ & $25 / 05 / 98$ & 68 \\
\hline \multicolumn{4}{|l|}{ DSAZ $1.2 \mathrm{~m}$} \\
\hline PG $1520+525$ & PG 1159 & $06 / 07 / 98$ & 157 \\
\hline PG $1424+535$ & PG 1159 & $06 / 07 / 98$ & 68 \\
\hline \multirow[t]{2}{*}{ PG $1520+525$} & PG 1159 & $22 / 05 / 99$ & 494 \\
\hline & & $25 / 05 / 99$ & 200 \\
\hline \multirow[t]{4}{*}{ PG $1424+535$} & PG 1159 & $19 / 05 / 99$ & 312 \\
\hline & & $20 / 05 / 99$ & 210 \\
\hline & & $25 / 05 / 99$ & 250 \\
\hline & & $27 / 05 / 99$ & 200 \\
\hline HS $1517+7403$ & PG 1159 & $24 / 05 / 99$ & 250 \\
\hline \multirow[t]{2}{*}{ Abell 43} & PG 1159 & $23 / 05 / 99$ & 1000 \\
\hline & & $27 / 05 / 99$ & 145 \\
\hline PG $1144+005$ & PG 1159 & $21 / 05 / 99$ & 250 \\
\hline \multirow[t]{2}{*}{ PG $1543+629$} & sdB & $27 / 05 / 99$ & 250 \\
\hline & & $29 / 05 / 99$ & 50 \\
\hline PG $1618+563$ & $\mathrm{sdB}$ & $26 / 05 / 99$ & 213 \\
\hline PG $1649+356$ & $\mathrm{sdB}$ & $24 / 05 / 99$ & 250 \\
\hline HS $1741+2133$ & $\operatorname{sdB}$ & $21 / 05 / 99$ & 250 \\
\hline HS $1831+6432$ & $\mathrm{sdB}$ & $24 / 05 / 99$ & 340 \\
\hline HS $1806+5024$ & $\operatorname{sdB}$ & $25 / 05 / 99$ & 240 \\
\hline HS $2100+1710$ & sdB & $21 / 05 / 99$ & 250 \\
\hline HS $2144+2231$ & $\mathrm{sdB}$ & $20 / 05 / 99$ & 200 \\
\hline HS $2202+2101$ & sdB & $22 / 05 / 99$ & 250 \\
\hline HS $2242+3206$ & sdB & $21 / 05 / 99$ & 250 \\
\hline
\end{tabular}

Reduction applies the bias and flatfield correction to the images and finds the exact position of the objects on the frame, which can shift for example due to tracking instabilities. The flux distribution is fitted with a gaussian, allowing the determination of its center to less-than-one-pixel accuracy.

Making the extraction mask involves marking the object, the reference stars and numerous fields of sky, a now highly automated yet very flexible process. 
Flux extraction considers the previously found position shifts to overlay the mask onto each image. Circular apertures are used to integrate the flux received from the object. This method is called (unweighted) aperture photometry, and it implies that the Gaussian fitted for finding the position of the object is not used here again. Rectangular patches of sky in the vicinity of the object provide a median value for the background subtraction.

Relative flux calculation: The background corrected flux is then related to one of the reference stars which have undergone the same procedure or, alternatively, to a super-reference star constructed from up to 10 individual reference stars, which increases the $\mathrm{S} / \mathrm{N}$ significantly. This can be done within a whole range of extraction radii and for various combinations of the possible reference stars without requiring significant $\mathrm{CPU}$ time.

The reduced data is the lightcurve for a chosen extraction radius and set of reference stars, i.e., each of the original frames is now reduced to one data point.

\subsection{Data analysis}

The following analysis first normalizes the data set to its median value, then cleans it from points with runaway flux values (which can happen when for example the centering procedure didn't work properly for a frame) and finally removes trends from the remaining set by fitting a polynomial of low degree. This set is once more being cleaned from runaway values with usually stricter upper and lower limits this time.

The search for periods: SCARGLE is a period search program handling unevenly spaced data points. It has been implemented by J. Wilms using a method described by Scargle (1982). Detections are controlled by confidence levels for the relative power through false alarm probabilities (fap). Fitting one or several sinus functions with iterative redetermination of their frequencies, amplitudes and phases has been implemented, too, and will be refined through the usage of PIKAIA, a genetic algorithm by Charbonneau (1995) which is to evolve towards the best combination of parameters. We also plan to do further checking by statistically rearranging and reanalyzing the data following Ciardullo \& Bond (1996).

The reduction of photometric CCD data in IDL is a fast and userfriendly implementation. This makes it suitable for final reduction 
right at the telescope. So next time you go observing, why don't you take TRIPP on the trip?

\subsection{Standard data set: analysis and results}

In order to check the quality of our photometry package TRIPP we reduced a data set kindly provided by $\mathrm{D}$. O'Donoghue obtained at the SAAO (South Africa) $1.9 \mathrm{~m}$ telescope with the UCT CCD photometer during the WET run in April 1999. We did not apply bias or flatfield corrections to the images provided. We used an aperture radius of 5.5 pixels for the star and 6 rectangular fields of sky with a side length of 9 pixels. The "standard" solution was obtained with an aperture radius of 5 pixels, with sky being measured in an annulus with inner and outer radii of 8 and 11 pixels, respectively. Also, we omitted all cleaning and fitting procedures described as part of the data analysis above. In Fig. 1 we compare our reduction to that obtained by A. Kanaan using IRAF. Our reduction is nearly as good as the IRAF version, proving its reliability. The scatter in our light curve is slightly higher in some parts, but better in other parts. More reduction packages are compared in the article of O'Donoghue in these proceedings.

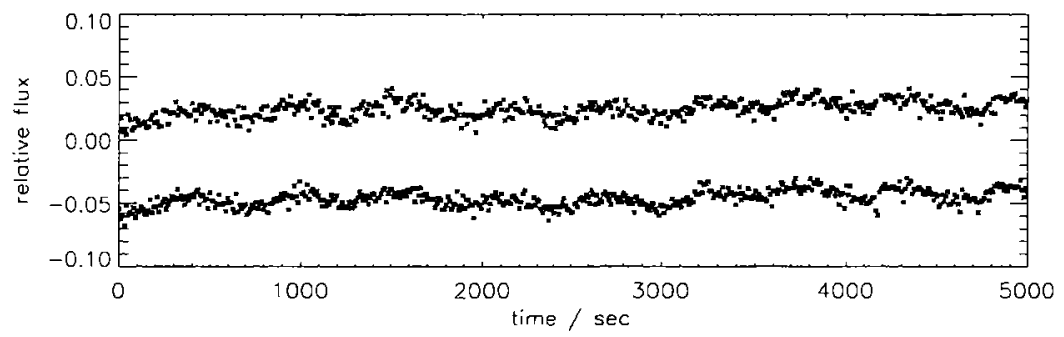

Fig. 1. Light curve of BPM 37093 extracted by TRIPP (top) compared to the one obtained by O'Donoghue \& Kanaan (bottom).

\section{RESULTS}

\subsection{Detected periods}

Table 2 shows the detected periods for our sample and for the XCOV17 objects in comparison to the known periods. We recover 
the major known periods and detect or suspect one new GW Vir and three EC 14026 variables.

The numbers in parentheses are $(1-$ fap $)$ in $\%$.

\begin{tabular}{|c|c|c|c|}
\hline Object & Known & $\begin{array}{l}\text { Detected } \\
\text { periods }(s)\end{array}$ & Suspected \\
\hline $\begin{array}{l}\text { PG } 1159-035 \\
\text { (WET object) }\end{array}$ & $\begin{array}{l}560,558,557 \\
518,517,516 \\
452,451,450 \\
416 \\
386 \\
364,363,362 \\
\text { (Winget et al. 1991) }\end{array}$ & $\begin{array}{l}562 \\
519\end{array}$ & $\begin{array}{l}517 \\
451 \\
415 \\
386 \\
362\end{array}$ \\
\hline Abell 43 & & $5500(99 \%)$ & \\
\hline $\begin{array}{l}\text { PG } 1336-018 \\
\text { (WET object) }\end{array}$ & $\begin{array}{l}186,185,184 \\
179,178,174 \\
141,135 \\
\text { (XCOV17; Kilkenny et al }\end{array}$ & $\begin{array}{l}179,174 \\
1998)\end{array}$ & $\begin{array}{r}186,184,182 \\
135\end{array}$ \\
\hline $\begin{array}{l}\text { PG } 1618+563 \\
\text { HS } 1806+5024 \\
\text { HS } 2242+3206 \\
\text { Feige } 48 \\
\text { (WET object) }\end{array}$ & $\begin{array}{l}379 \text { (Koen et al. 1998) } \\
351,348,343\end{array}$ & $\begin{array}{l}379 \\
346\end{array}$ & $\begin{array}{l}522(80 \%) \\
127(80 \%)\end{array}$ \\
\hline
\end{tabular}

\subsection{Comments on some specific results}

A43: While Ciardullo \& Bond (1996) found a possible period of 41.2 minutes but hesitate to claim variability of this star because the period did not pass their strict criteria, we detect variability on an even longer scale $(5500 \mathrm{~s})$. This is the longest known period for a PG 1159 star; HS 2324+3944 (Silvotti et al. 1999), a similar hybrid PG 1159 star, displays its strongest period at $2100 \mathrm{~s}$. Whether this is the orbital period of a binary system or a pulsation mode remains to be clarified, but the argument presented below supports the reality of this detection. Simulating a theoretical lightcurve that contains the $5500 \mathrm{~s}$ period but of shorter length than our run (namely $3.42 \mathrm{~h}$, the length of Ciadullo \& Bond's data set), the subsequent analysis yields an artificial yet significant detection around $2400 \mathrm{~s}$. 
PG 1618+563: An early detection of variability was not confirmed by our confidence check. However, Silvotti et al. (see these Proceedings) detected a close doublet at $140 / 144 \mathrm{~s}$ with the Nordic Optical Telescope (NOT).

\section{ACKNOWLEDGMENTS}

Thanks to G. Lamer for the July 1998 data and to T. Rauch for participation in the May 1999 observing run. This work is based on observations collected at the German-Spanish Astronomical Center (DSAZ), Calar Alto, operated by the Max-Planck-Institut für Astronomie, Heidelberg jointly with the Spanish National Commission for Astronomy. Several travel grants to the Calar Alto Observatory were provided by the DFG. This work was supported by NATO ARW and IITAP (UNESCO) funds.

\section{REFERENCES}

Charbonneau P. 1995, ApJS, 101, 309

Ciardullo R., Bond H. E. 1996, AJ, 111, 2332

Heber U., Edelmann H., Lemke M., Napiwotzki R., Engels D. 1999 in 11th European Workshop on White Dwarfs, eds. J.-E.Solheim \& E. G. Meištas, ASP Conf. Ser., 169, 551

Kilkenny D., O'Donoghue D., Koen C., Stobie R.S. 1997, MNRAS, 285, 640

Kilkenny D., O'Donoghue D., Koen C., Lynas-Gray A. E., van Wyk F. 1998, MNRAS, 296, 329

Koen C., O'Donoghue D., Pollacco D. L., Nitta A. 1998, MNRAS, 300, 1105 Scargle J. D. 1982, ApJ, 263, 835

Silvotti R., Dreizler S., Handler G., Jiang X. J. 1999, A\&A, 342, 745

Winget D. E., Nather R. E., Clemens J. C. et al. 1991, ApJ, 378, 326 